\title{
Monitoring Microfluidic Interfacial Flows Using Impedance Spectroscopy
}

Nicholas Mavrogiannis ${ }^{a}$, Xiaotong $\mathrm{Fu}^{\mathrm{a}}$, Mitchell Desmond ${ }^{\mathrm{a}}$, Robert McLarnon ${ }^{\mathrm{a}}$, Zachary Gagnon $^{\text {a* }}$

aDepartment of Chemical and Biomolecular Engineering, Johns Hopkins University, Maryland Hall 220A, Baltimore MD, 21218

*Corresponding Author. zgagnon1@jhmi.edu 


\section{Abstract}

Microfluidic platforms capable of complex on-chip processing and liquid handling enable a wide variety of sensing, cellular, and material-related applications across a spectrum of disciplines in engineering and biology. However, there is a general lack of available microscale sensors capable of non-optically monitoring and quantifying on-chip fluid motion. Hence, many microfluidic systems are confined to the laboratory because their use requires optical microscopy. Here, we present a method for dynamically tracking laminar interfacial flows in microfluidic channels non-optically using impedance spectroscopy. Using a microfluidic T-channel, we generate a liquid interface by coflowing two different electrolyte streams side-by-side. The interface is driven through an array of "displacement" electrodes where it is electrokinetically deflected across the microchannel. The interfacial flow is monitored downstream using an array of interdigitated "impedance" electrodes which dynamically measure the electrochemical impedance near the surface of the flow channel. We demonstrate that the impedance spectrum is sensitively influenced by the position of the deflected interface. While laminar fluid interfaces are ubiquitous to microfluidic flows and used extensively in rheology and biomolecular detection, it is currently difficult to measure their position non-optically. The sensing method presented here enables the interfacial position to be dynamically determined without a microscope and provides a new tool for lowering the barriers to operating microfluidic devices outside the confines of a traditional laboratory.

Keywords: Electrical Impedance Spectroscopy, Microfluidics, Liquid Interface, Fluidic Dielectrophoresis 


\section{Introduction}

Microfluidics offers an attractive platform for performing miniaturized chemical and biomolecular analysis. Particularly useful is the ability to embed multiple laboratory steps, including preparation and chemical detection, into a microfluidic chip for automated sample processing and analysis. An important engineering design challenge in microfluidics is to develop new in situ analytical tools that monitor these operations within the confines of the microchannel network. Fluorescent-microscopy, for example, has been used to perform noninvasive quantification of liquid properties and flow velocity in microchannels [1]. Other techniques such as in situ Ramen spectroscopy [2], imaging FTIR [3] and cyclic voltammetry [4] have been used to capture microscale chemical images of microfluidic channel surfaces and fluidic flow patterns. Another strategy relies on employing electrochemical impedance spectroscopy (EIS) to monitor electrical changes on microfluidic surfaces [5] and cells [6] in contact with electrolyte. Because EIS can be performed with micro-electrodes integrated on-chip, it has potential to be a powerful tool for rapid non-optical monitoring of microfluidic processes.

EIS is used for monitoring complex surface processes in microfluidic space without the use of fluorescent labels and fixatives. By detecting variations in impedance as a function of field frequency, EIS, for example, can be used to non-optically detect proteins $[7,8]$ and DNA $[9,10]$ through monitoring protein binding and DNA hybridization on electrode surfaces, where these interactions influence current flow and the corresponding impedance spectrum $[17,18]$. EIS can also be used to quantify cell growth [11,12]. Cells, for example can be grown on the surface of patterned EIS microelectrodes [13]. The electrode impedance has been shown to be influenced by the cell type and cell state [14-16].

Because EIS can be easily integrated into microfluidic flow channels, its utility could be extended to non-optically monitoring fluidic behavior for monitoring on-chip mixing [19] and routing operations [20] where multiple fluid streams are routed, mixed, and pumped across a network of microchannels. In this work, we demonstrate how to 
use EIS for non-optically monitoring fluid motion produced by an electrokinetic flow. One feature of low Reynolds number $(R e)$ flows is that multiple liquid streams can flow sideby-side without convectively mixing. The result is that well-controlled microfluidic laminar interfaces can be created between both miscible and immiscible liquids. Interfacial flows are ubiquitous in low Re systems and play important roles in microfluidic applications in rheology [21], chemical detection [22], molecular mass sensors [23], immunoassays [24], DNA hybridization [25], and kinetic analysis [26,27].

Recently, we reported a new electrokinetic method for manipulating these liquid interfaces [28]. The technique was demonstrated using two fluids with different electrical properties driven side-by-side in a microfluidic channel. When an alternating current (AC) electric field was applied perpendicular to the direction of flow, the laminar interface polarized and the fluid electrohydrodynamically displaced across the flow channel. The motion of the fluid interface was shown to be an effective electrokinetic method for assessing fluid electrical conductivity and dielectric constant [29], and for detecting biomolecular binding [30]. In order to perform these fluidic measurements, however, the liquid interface required constant optical monitoring with confocal microscopy. While, effective, a non-optical approach could offer a smaller, less costly and potentially automated means to perform interfacial measurements.

In this work, we present a non-optical EIS technique for dynamically tracking the position of a miscible microfluidic liquid interface under electrokinetic flow. Our method utilizes two different electrode arrays: an upstream parallel point electrode array to polarize and induce electrokinetic flow at a liquid interface and a downstream series of interdigitated electrodes to dynamically measure the EIS on the microchannel surface. We show that the magnitude of the impedance $(|Z|)$ is sensitive to the position of the liquid interface and can be used to track interfacial motion in response to electrokinetically-induced fluid flow. We propose that variations of this system may be useful for non-optically monitoring the dynamics of microfluidic interfaces in electrokinetics, rheology, biomolecular detection, and microfluidic mixing applications. 
We begin by describing electrokinetic flow and interfacial displacement (sec 2.1). We then describe the experimental strategy for producing these flows (sec 2.2-2.3) and the experimental setup used to dynamically measure impedance (2.4). Finally, sec. 3 presents impedance results obtained for a range of electric field frequencies and interfacial positions.

\section{Experimental}

\subsection{Electrokinetic Flow}

AC electric fields have long been exploited to manipulate the fluidic contents of microfluidic systems. When an electric field is applied across a charged surface or within a non-neutral liquid domain, an electrical stress is produced which can drive fluid flow. In liquids, these electrokinetic flows occur when a field is applied tangential to a charged surface (e.g electro-osmosis) or across regions of fluid with spatial gradients in electrical properties (e.g electrothermal flow). Recently, a new type of electrokinetic flow was discovered in the vicinity of laminar liquid interfaces formed between two miscible aqueous electrolytes [28]. The phenomenon is known as fluidic dielectrophoresis (fDEP), which describes the ability to deflect a laminar liquid interface across a microchannel using an external AC electric field [29]. The interface is created using a microfluidic T-channel device where two fluids are forced to flow side-by-side. Each liquid has a different electrical conductivity $(\sigma)$ and dielectric constant $(\varepsilon)$ such that a large electrical mismatch exists at their interface. fDEP motion is created using a perpendicular electric field produced from an array of parallel point electrodes integrated on the surface of the microfluidic channel (Fig 1a). For a liquid interface subjected to a time varying monochromatic electric field, the displacement is directly proportional to the real part of the interface polarizability factor, $K(\omega)$, which is function of field frequency $(\omega)$, electrical conductivity and permittivity [29]:

$\operatorname{Re}[K(\omega)]=\frac{\left(\varepsilon_{2}-\varepsilon_{1}\right) \tau^{2} \omega^{2}}{\left(\varepsilon_{2}+\varepsilon_{1}\right)\left(\tau^{2} \omega^{2}+1\right)}+\frac{\left(\sigma_{2}-\sigma_{1}\right) \tau^{2} \omega^{2}}{\left(\sigma_{2}+\sigma_{1}\right)\left(\tau^{2} \omega^{2}+1\right)}$, 
where $\tau=\left(\frac{\varepsilon_{2}+\varepsilon_{1}}{\sigma_{2}+\sigma_{1}}\right)$ is the characteristic Maxwell-Wagner charge relaxation timescale at the interface between the two liquids. As depicted in Eq. (1), because the interfacial polarization (e.g. charging) is driven by both conductive and dielectric charging, displacement is a function of the electrical properties of each fluid stream and the AC electric field frequency. At frequencies on the order of $100 \mathrm{kHz}$, for example, the magnitude of interface displacement is governed solely by differences in the electrical conductivity between the two co-flowing fluids, which we define here as the interfacial conductivity $\left(\sigma_{2}-\sigma_{1}\right)$. At high frequency (typically $>10 \mathrm{MHz}$ ), however, the displacement is driven by the interfacial permittivity $\left(\varepsilon_{2}-\varepsilon_{1}\right)$. Finally, at intermediate frequency the interface behavior is sensitive to both electrical and dielectric differences. Because of the low and high frequency attributes of fDEP, if one fluid phase has a greater conductivity $\left(\sigma_{1}>\sigma_{2}\right)$ and the adjacent fluid has a greater dielectric constant $\left(\varepsilon_{2}>\varepsilon_{1}\right)$, the direction of the interface displacement will reverse at a critical frequency and there will be a characteristic cross-over frequency (COF) where no interface displacement is observed. This occurs when the interface's polarizability factor is zero, which can be expressed as [29]:

$\omega_{C O F}=\frac{1}{2 \pi}\left[\frac{\left(\sigma_{1}-\sigma_{2}\right)\left(\sigma_{1}+\sigma_{2}\right)}{\left(\varepsilon_{2}-\varepsilon_{1}\right)\left(\varepsilon_{2}+\varepsilon_{1}\right)}\right]^{1 / 2}$.

At low frequency below the COF, the high conductive fluid displaces across the flow channel (Fig 1b). At the COF the net charge on the interface is zero and no displacement is observed (Fig 1c). Finally, above the COF the interface displaces in the opposite direction (Fig 1d) and the low conductive high dielectric fluid displaces across the channel. We next discuss the experimental setup for measuring the displacement direction and magnitude non-optically using EIS.

\subsection{Design and Fabrication of Microfluidic Device with Embedded Electrokinetic and Impedance Electrodes}

The experimental design requires a laminar interface and two different types of 
electrode arrays to displace and subsequently detect the interface position. We used a microfluidic "T-channel" device to create the liquid interface. Two fluid streams were supplied to the microfluidic device using a low-cost constant pressure source flow system [31]. The microfluidic device was fabricated using standard soft photolithography and microfabrication techniques. Microchannel electrodes were fabricated using wet chemical etching. Glass cover slips (50×30 mm, no.1, Fisher Scientific) were coated with $2 \mathrm{~nm}$ of chromium and $50 \mathrm{~nm}$ of gold using electron beam evaporation. The cover slips were patterned with photoresist (Shipley 1813) and exposed metal was etched using gold and chromium etchant. The resulting electrode pattern was then aligned and bonded to a soft lithographically fabricated T-channel device. To fabricate this device, the "T-channel" pattern was lithographically fabricated to a silica wafer using SU-8 3050 photoresist (Microchem Corp.). A 10:1 mixture of polydimethylsiloxane (PDMS) elastomer and curing agent was poured atop the wafer and baked at $85{ }^{\circ} \mathrm{C}$ for 30 minutes. The PDMS was gently peeled off the wafer and cut out of the mold. Fluid ports were punched with a $0.75 \mathrm{~mm}$ diameter biopsy punch (Ted Pella, Inc.). The electrode patterned coverslip was then exposed to oxygen plasma (Jelight, Model 42A), the PDMS microchannel was exposed using a handheld tesla coil (Electro-Technic Products Inc. Model BD-20) and the two substrates were immediately aligned and sealed under an inverted microscope. The assembled device consisted of a main flow channel $150 \mu \mathrm{m}$ in width and $65 \mu \mathrm{m}$ in height with an upstream displacement (parallelpoint) and a downstream impedance $\left(45^{\circ}\right.$-interdigitated) electrode array (Fig 1). To perform an experiment, the fluid interface was subjected to an electric field using the parallel-point electrode array and forced to displace by fDEP at different field frequencies (Fig 1b-d). As fluid exited the first displacement array, the interfacial stress ceased. Because the inertial influence on the flow is minimal $(R e<1)$, the fluid interface remained fixed in a displaced position immediately after exiting the fDEP array. We then determined the deflection position by measuring the magnitude of the impedance using a second array of interdigitated electrodes.

\subsection{Chemicals and Reagents}


The liquid interface was composed of two fluids, each with a different electrical conductivity $(\sigma)$ and dielectric constant $(\varepsilon)$. When forced to flow side-by-side at low Reynolds number these two fluids formed an interface with a large electrical mismatch between them. Each stream was injected at a constant flow rate $(10 \mu \mathrm{L} / \mathrm{min})$ into the device using a low-cost flow controller equipped with an externally pressurized fluidfilled cryogenic vial [31]. We labelled each fluid with a different Alexa Fluor fluorescent dye to accurately image the interface position using confocal microscopy. Shown in Fig 1, the electrical interface was formed by flowing a left-most (green) 1x PBS solution $\left(\sigma_{1}=0.29 \mathrm{mS} / \mathrm{cm} ; \varepsilon_{1}=78\right)$ with $10 \mathrm{ng} / \mathrm{mL}$ of Alexa Fluor 488 (Invitrogen). The rightmost (red) high dielectric stream $\left(\sigma_{2}=19 \mu \mathrm{S} / \mathrm{cm} ; \varepsilon_{2}=110\right)$ was comprised of 0.8M 6aminohexanoic acid (Sigma-Aldrich) (AHA) labeled with $10 \mathrm{ng} / \mathrm{mL}$ of Alexa Fluor 594 (Invitrogen). AHA is a water-soluble zwitterion used for increasing the dielectric constant of aqueous solution [28]. Prior to fluorescent labeling, the AHA solution was polished with $1 \mathrm{~g} / \mathrm{mL}$ Dowex MR-3 (Sigma) ion exchange resin to remove trace salts and reduce solution conductivity. The COF of this electrolyte system was measured using previously published methods [29] and found to be $6.2 \mathrm{MHz}$.

\subsection{Impedance Measurement}

We utilized the upstream parallel-point array to drive fDEP flow across the channel and a second downstream $45^{\circ}$-interdigitated array as an impedance sensor. The parallel-point electrodes were axially separated by $20 \mu \mathrm{m}$ and symmetrically bridged the width of the microchannel. We used electrodes with sharp points to focus the electric field to the tip of the electrodes and to provide increased contact with the PDMS and glass substrate along the main flow channel walls. A function generator (Rigol DG4102) was connected to the fDEP electrodes and delivered an AC electric field to displace the interface across the channel. The downstream impedance electrodes were interdigitated and positioned at a $45^{\circ}$ angle relative to the flow direction to maximize the sensitivity of the array to changes in interfacial position. An impedance spectrometer (Sciospec ISX-5) was connected to the impedance electrode array and used to measure the magnitude of the impedance as a function of interface position. For 
all impedance measurements, a sine-modulated AC potential of $50 \mathrm{mV}$ was applied to the electrode array and the magnitude and phase angle of impedance were measured over an excitation frequency range between $100 \mathrm{kHz}$ to $10 \mathrm{MHz}$.

\section{Results and discussion}

\subsection{Imaging Interfacial Position During Electrokinetic Displacement}

Fig $1 \mathrm{~b}$-d depicts a top-view of the interfacial motion of the interface over the length of the fDEP electrode array when a 10-volt peak-to-peak $\left(V_{p p}\right)$ potential was applied across the displacement electrodes at three different field frequencies $(1 \mathrm{MHz}, 6.2 \mathrm{MHz}$, and $20 \mathrm{MHz}$ ). When the electric field frequency was $1 \mathrm{MHz}$, the conductive PBS (green) stream displaced across the interface (Fig 1b). When the COF $(6.2 \mathrm{MHz})$ was applied, both conductive and dielectric forces were equal, and the interface remained fixed as it passed through the electrode array (Fig 1c). Finally, at a frequency above the COF (20 $\mathrm{MHz}$ ) the deflection reversed direction and the high dielectric stream (red) displaced across the microchannel (Fig 1d).

\subsection{Characterization of Electrokinetic Interfacial Flow Using Impedance Spectroscopy}

Because the interfacial-driven motion of the fluid ceases upon exiting the displacement electrode array, the interface's displaced position can be accurately determined using the downstream impedance electrode array (Fig 2). The top-view micrographs shown in Fig 2a-c illustrate that when the interface was subjected to either a low or a high frequency electric field, a greater degree of either high conductive-low dielectric PBS or low conductive-high dielectric AHA covered the sensing electrodes at low and high field frequency, respectively. To observe the 3D structure of the interfacial flow field, 2D confocal micrographs were captured above the impedance electrode array for three different electric field frequencies: 1, 6.2 and $20 \mathrm{MHz}$ (Fig 2) Since the displacement electrodes are thin co-planar films $(\sim 52 \mathrm{~nm})$ and confined to the microchannel surface, the electrical stress responsible for driving flow was localized 
near the surface of the microchannel. In order to satisfy mass conservation, this local electrokinetic flow was countered by a pressure driven back flow at the top of the channel which produced a "tilted" interface, as shown in the 2D confocal micrographs in Fig 2. Because the impedance electrode array is also confined to the microchannel surface, the impedance measurements were only sensitive to the electrical properties of the fluid domain very near the surface where the electric field was capable of penetrating into the liquid domain. Therefore, differences in interfacial position produced by fDEP will create changes in both the local conductivity and dielectric constant of the fluid near the impedance sensor.

In order to determine the optimum impedance conditions for measuring interfacial position, we first determined how upstream fDEP displacement influences downstream impedance over a range of impedance excitation frequencies. An electrical interface was created by co-flowing solutions of PBS and AHA, and then deflected at frequencies below $(1 \mathrm{MHz})$ and above $(20 \mathrm{MHz})$ the COF, and when no field was applied (e.g. the position at the COF). For each interface position, we performed an impedance frequency sweep from $100 \mathrm{kHz}$ to $5 \mathrm{MHz}$ to determine the magnitude of impedance for different interfacial positions (Fig 3). Below the interfacial COF at $1 \mathrm{MHz}$, the fDEP electrodes polarized and forced the high conductive (green) stream to cover a larger area on the impedance electrode array. Conversely, when we applied a high frequency above the COF, the high dielectric stream displaced across the interface and the impedance sensor was exposed to fluid with lower electrical conductivity. The impedance data was consistent with the electrical changes that the interface position produces in the vicinity of the impedance electrodes. When high conductive-low dielectric PBS covered a greater portion of the impedance electrode array, the impedance decreased, while the opposite was seen when the low conductive-high dielectric AHA stream displaced across the impedance electrode array. Because the interface position is not influenced by the electric field at the COF, the magnitude of the impedance at the COF is identical the case when no displacement field was applied since the interface does not displace at the COF. Shown in Fig 3, the interface deflection produced the greatest change in the magnitude of the impedance $(|Z|)$ at an 
impedance frequency of $\sim 500 \mathrm{kHz}$. Based on these experiments, we measured $|\mathrm{Z}|$ at a frequency of $500 \mathrm{kHz}$ for all subsequent experiments in this work.

\subsection{Determining Interface Position Using Impedance Spectroscopy}

With the impedance excitation frequency fixed at $500 \mathrm{kHz}$, we next measured $|\mathrm{Z}|$ as a function interface position for three different applied voltages $\left(5 V_{p p}, 10 V_{p p}\right.$ and 15 $\mathrm{V}_{\mathrm{pp}}$ ) delivered across the interface using the upstream displacement electrode array. For each applied voltage the fDEP frequency was continuously swept from $1-20 \mathrm{MHz}$, and then back to $1 \mathrm{MHz}$ while simultaneously measuring $|\mathrm{Z}|$ at the downstream impedance array. Shown in Fig 4, when the interface is centered at the COF, $|Z|$ was found to be $32.5 \mathrm{k} \Omega$ for all three voltages applied. However, when an fDEP frequency below the COF was applied, the high conductive PBS stream covered a larger portion of the impedance sensor surface and the impedance decreased from $25 \mathrm{k} \Omega$ to $15 \mathrm{k} \Omega$ at an applied voltage of $5 \mathrm{~V}_{\mathrm{pp}}$. When the fDEP frequency increased above the COF, the high dielectric fluid covered a greater portion of the sensor surface and $|Z|$ increased to 45 $\mathrm{k} \Omega$. The impedance was also influenced when the electrical interface was subjected to larger displacement voltages. This increase was particularly noticeable at high

frequencies (> COF) where more low conductive, high dielectric AHA buffer covered a greater area of the impedance sensor. In order to visualize the interface, we captured $3 \mathrm{D}$ confocal micrographs of the interface when a $20 \mathrm{MHz}$ AC electric field was applied at voltages of 5,10 and $15 \mathrm{Vpp}$. Shown in the micrographs in Fig 4, the AHA stream (read) covers a greater electrode area with increasing applied voltage. While there is a large change in impedance with increasing voltage at high frequencies, the three $|\mathrm{Z}|$ datasets are not as strongly influenced at low frequencies (<COF). This is because changes in the position of the high conductivity buffer on the impedance electrodes do not influence the impedance to the same magnitude as the AHA buffer. Lastly, when the interface COF was applied to the interface, the interface did not displace in the electric field, and therefore the impedance did not change with applied voltage.

Next, we determined if it was possible to distinguish the COF between two fluid 
interfaces with different electrical conductivity mismatches. In Fig 5, we show impedance results for two different interfacial conductivities - one where $\Delta \sigma=\sigma_{2}-\sigma_{1}=$ 0.27 and a second interface where $\Delta \sigma=0.61 \mathrm{mS} / \mathrm{cm}$. Shown in Fig 5 , we observed a difference in measured impedance for the two different interfaces, where the impedance measurements for a given interface position increased with interfacial conductivity. For an alternative way to analyze these results, we normalized the impedance of each dataset by the initial impedance value when no electric field was applied using a timeaveraged baseline impedance reading that was taken prior to applying an electric field at the displacement electrodes. Shown in Fig 5b, we see that both fluidic systems begin and end at the same dimensionless impedance, $|Z|^{*}$, but their COF's are different and increase with interfacial conductivity, as is consistent with previous fDEP experiments [29].

\section{Conclusions}

In this paper, we presented a novel method for detecting an electrokinetically displaced liquid interface using impedance spectroscopy. Using an AC electric field, we forced a laminar fluid interface to deflect across a microchannel using fDEP and then measured the position of the deflected interface using a downstream impedance electrode array. The interface was composed of two co-flowing fluids - one stream had a greater electrical conductivity and the other had a greater dielectric constant. When the interface was exposed to a low frequency $A C$ electric field, high conductive fluid stream displaced across the channel and covered a larger surface area of the impedance sensor, which reduced the magnitude of the impedance. As the frequency was increased to the COF, the interfacial position moved to its original position which reduced the amount of conductive fluid over the impedance array. At higher electric field frequencies above this COF, the interfacial deflection reversed direction and the high dielectric, low conductive stream covered a larger surface of the impedance sensor and produced an increase in impedance. This method was able to electrically detect interfacial displacement and measure the interface COF non-optically at a resolution consistent with experiments performed with confocal microscopy. We believe this 
method provides a new sensing technique for non-optically imaging microfluidic flow fields. While successful at detecting motion induced by electrokinetic flows, we observed that hydraulic-induced changes in interfacial position through increased flow rate also influenced the impendence measurements. Future work will focus on developing an equivalent circuit model to account for this influence and to accurately predict the limit of detection for which interface motion can be non-optically measured.

\section{Acknowledgements}

We gratefully acknowledge support from the National Science Foundation under CBET CAREER grant 1351253 and CBET grant 1511185. 


\section{References}

[1] A. Günther, K.F. Jensen, Multiphase microfluidics: from flow characteristics to chemical and materials synthesis, Lab Chip. 6 (2006) 1487-1503. doi:10.1039/B609851G.

[2] K. Kim, H.S. Han, I. Choi, C. Lee, S. Hong, S.-H. Suh, et al., Interfacial liquidstate surface-enhanced Raman spectroscopy, Nature Communications. 4 (2013) 2182. doi:10.1038/ncomms3182.

[3] K.L.A. Chan, S. Gulati, J.B. Edel, A.J. de Mello, S.G. Kazarian, Chemical imaging of microfluidic flows using ATR-FTIR spectroscopy, Lab Chip. 9 (2009) 2909-2913. doi:10.1039/b909573j.

[4] J.M. Salverda, A.V. Patil, G. Mizzon, S. Kuznetsova, G. Zauner, N. Akkilic, et al., Fluorescent Cyclic Voltammetry of Immobilized Azurin: Direct Observation of Thermodynamic and Kinetic Heterogeneity, Angewandte Chemie. 122 (2010) 5912-5915. doi:10.1002/ange.201001298.

[5] S.L. Wu, Z.D. Cui, G.X. Zhao, M.L. Yan, S.L. Zhu, X.J. Yang, EIS study of the surface film on the surface of carbon steel from supercritical carbon dioxide corrosion, Applied Surface Science. 228 (2004) 17-25.

doi:10.1016/j.apsusc.2003.12.025.

[6] C. Xiao, J.H.T. Luong, On-line monitoring of cell growth and cytotoxicity using electric cell-substrate impedance sensing (ECIS), Biotechnol. Prog. 19 (2003) 1000-1005. doi:10.1021/bp025733x.

[7] J.A. Lee, S. Hwang, J. Kwak, S.I. Park, S.S. Lee, K.-C. Lee, An electrochemical impedance biosensor with aptamer-modified pyrolyzed carbon electrode for label-free protein detection, Sensors and Actuators B: Chemical. 129 (2008) 372-379. doi:10.1016/j.snb.2007.08.034.

[8] H. Cai, T.M.-H. Lee, I.-M. Hsing, Label-free protein recognition using an aptamer-based impedance measurement assay, Sensors and Actuators B: Chemical. 114 (2006) 433-437. doi:10.1016/j.snb.2005.06.017.

[9] A.A. Ensafi, M. Taei, H.R. Rahmani, T. Khayamian, Sensitive DNA impedance biosensor for detection of cancer, chronic lymphocytic leukemia, based on gold nanoparticles/gold modified electrode, Electrochimica Acta. 56 (2011) 81768183. doi:10.1016/j.electacta.2011.05.124.

[10] Y. Hu, K. Wang, Q. Zhang, F. Li, T. Wu, L. Niu, Decorated graphene sheets for label-free DNA impedance biosensing, Biomaterials. 33 (2012) 1097-1106. doi:10.1016/j.biomaterials.2011.10.045.

[11] S.C.C. Shih, I. Barbulovic-Nad, X. Yang, R. Fobel, A.R. Wheeler, Digital microfluidics with impedance sensing for integrated cell culture andanalysis, Biosens Bioelectron. 42 (2013) 314-320. doi:10.1016/j.bios.2012.10.035.

[12] S. Reitinger, J. Wissenwasser, W. Kapferer, R. Heer, G. Lepperdinger, Electric impedance sensing in cell-substrates for rapid and selective multipotential differentiation capacity monitoring of human mesenchymal stem cells, Biosens Bioelectron. 34 (2012) 63-69. doi:10.1016/j.bios.2012.01.013.

[13] J. Wegener, C.R. Keese, I. Giaever, Electric Cell-Substrate Impedance Sensing (ECIS) as a Noninvasive Means to Monitor the Kinetics of Cell Spreading to 
Artificial Surfaces, Exp. Cell Res. 259 (2000) 158-166.

doi:10.1006/excr.2000.4919.

[14] T.A. Nguyen, T.-I. Yin, D. Reyes, G.A. Urban, Microfluidic chip with integrated electrical cell-impedance sensing for monitoring single cancer cell migration in three-dimensional matrixes, Anal. Chem. 85 (2013) 11068-11076. doi:10.1021/ac402761s.

[15] J.-L. Hong, K.-C. Lan, L.-S. Jang, Electrical characteristics analysis of various cancer cells using a microfluidic device based on single-cell impedance measurement, Sensors and Actuators B: Chemical. 173 (2012) 927-934. doi:10.1016/j.snb.2012.06.046.

[16] S. Michaelis, J. Wegener, R. Robelek, Label-free monitoring of cell-based assays: combining impedance analysis with SPR for multiparametric cell profiling, Biosens Bioelectron. 49 (2013) 63-70. doi:10.1016/j.bios.2013.04.042.

[17] Y. Xu, Y. Jiang, H. Cai, P.-G. He, Y.-Z. Fang, Electrochemical impedance detection of DNA hybridization based on the formation of M-DNA on polypyrrole/carbon nanotube modified electrode, Analytica Chimica Acta. 516 (2004) 19-27. doi:10.1016/j.aca.2004.04.013.

[18] Y. Hu, F. Li, X. Bai, D. Li, S. Hua, K. Wang, et al., Label-free electrochemical impedance sensing of DNA hybridization based on functionalized graphene sheets, Chem. Commun. 47 (2011) 1743-1745. doi:10.1039/C0CC04514D.

[19] T.J. Johnson, D. Ross, L.E. Locascio, Rapid microfluidic mixing, Anal. Chem. 74 (2002) 45-51.

[20] J.W. Kwon, E.S. Kim, Multi-level microfluidic channel routing with protected convex corners, Sensors and Actuators a: Physical. 97-98 (2002) 729-733. doi:10.1016/S0924-4247(02)00012-2.

[21] D.E. Solomon, S.A. Vanapalli, Multiplexed microfluidic viscometer for highthroughput complex fluid rheology, Microfluid Nanofluid. 16 (2013) 677-690. doi:10.1007/s10404-013-1261-2.

[22] A. Hatch, E. Garcia, P. Yager, Diffusion-based analysis of molecular interactions in microfluidic devices, Proceedings of the IEEE. 92 (2004) 126-139. doi:10.1109/JPROC.2003.820547.

[23] S. Mandal, J.M. Goddard, D. Erickson, A multiplexed optofluidic biomolecular sensor for low mass detection, Lab Chip. 9 (2009) 2924-2932. doi:10.1039/b907826f.

[24] A. Hatch, A.E. Kamholz, K.R. Hawkins, M.S. Munson, E.A. Schilling, B.H. Weigl, et al., A rapid diffusion immunoassay in a T-sensor, Nature Biotechnology. 19 (2001) 461-465. doi:10.1038/88135.

[25] X. Weng, H. Jiang, D. Li, Microfluidic DNA hybridization assays, Microfluid Nanofluid. 11 (2011) 367-383. doi:10.1007/s10404-011-0858-6.

[26] J.H.-S. Kim, A. Marafie, X.-Y. Jia, J.V. Zoval, M.J. Madou, Characterization of DNA hybridization kinetics in a microfluidic flow channel, Sensors and Actuators B: Chemical. 113 (2006) 281-289. doi:10.1016/j.snb.2005.03.034.

[27] H. Song, R.F. Ismagilov, Millisecond kinetics on a microfluidic chip using nanoliters of reagents, J. Am. Chem. Soc. 125 (2003) 14613-14619. doi:10.1021/ja0354566.

[28] M. Desmond, N. Mavrogiannis, Z. Gagnon, Maxwell-Wagner polarization and 
frequency-dependent injection at aqueous electrical interfaces, Phys. Rev. Lett. 109 (2012) 187602.

[29] N. Mavrogiannis, M. Desmond, Z.R. Gagnon, Fluidic dielectrophoresis: The polarization and displacement of electrical liquid interfaces, Electrophoresis. 36 (2015) 1386-1395. doi:10.1002/elps.201400454.

[30] N. Mavrogiannis, F. Crivellari, Z.R. Gagnon, Label-free biomolecular detection at electrically displaced liquid interfaces using interfacial electrokinetic transduction (IET), Biosens Bioelectron. 77 (2016) 790-798. doi:10.1016/j.bios.2015.10.045.

[31] N. Mavrogiannis, M. Ibo, X. Fu, F. Crivellari, Z. Gagnon, Microfluidics made easy: A robust low-cost constant pressure flow controller for engineers and cell biologists, Biomicrofluidics. 10 (2016) 034107. doi:10.1063/1.4950753. 


\section{Figure Captions}

Figure 1. Confocal micrograph with two embedded electrode arrays. (a) Two co-flowing fluids with varying electrical properties in are driven into a microfluidic t-channel device. Each inlet channel $(75 \mu \mathrm{m}$ in width) merges with a main $150 \mu \mathrm{m}$ wide main channel to create a sharp liquid interface. One stream has a larger conductivity (green), while the adjacent stream (red) has a large permittivity. The main channel has two embedded electrode arrays - displacement electrodes actuate the liquid electrical interface and sensing electrodes measures the local impedance. (b) $\mathrm{A} 1 \mathrm{MHz}$ ac electric field is applied to displacement electrodes, displacing the high conductive stream (green) into the low conductive stream (red) A dotted white line shows the original interface position when no electric field is applied. (c) At a COF of $6.2 \mathrm{MHz}$ the interface does not deflect in the field. (d) At $20 \mathrm{MHz}$ the high dielectric stream (red) displaces into the low dielectric stream (green).

Figure 2. Top-down confocal micrographs of the interface position taken above the impedance sensing electrodes for three different electric field frequencies applied the upstream displacement electrodes. A magnified 3D confocal z-stack is depicted below each top-down micrograph. For each image pair, a dotted white line highlights the interface position when no field is applied. (a) At a field frequency of $1 \mathrm{MHz}$ the high conductive stream (green) is driven across the channel surface. The corresponding 3D confocal stack shows that an increase in conductive green fluid covering the surface of the sensing electrodes. (b) The COF, 6.2 MHz, is applied and the liquid interface remains fixed. (c) The direction of displacement reverses at $20 \mathrm{MHz}$ and the high dielectric stream displaces into the low dielectric stream. The 3D image shows the direction reversed, leading to a decrease in conductive stream covering the impedance electrodes.

Figure 3. The magnitude of the impedance $|z|$ versus applied excitation frequency for different fDEP interface positions. Three frequency sweeps were performed in order to determine the optimal frequency for the impedance spectrometer. When $1 \mathrm{MHz}$ is applied to the displacement electrodes, the high conductive-low dielectric (green) stream covers more impedance sensor electrode area and the impedance magnitude decreases. When a $20 \mathrm{MHz}$ electric field is applied the high dielectric-low conductive (red) stream occupies a larger sensing electrode area and the impedance increases. The magnitude of impedance remains the same when the field is off and the COF is applied.

Figure 4. Magnitude of the impedance for different fDEP frequencies at an impedance excitation frequency of $500 \mathrm{kHz}$. The interface was displaced at 5,10 and $15 \mathrm{~V}_{\mathrm{pp}}$. As voltage increased, the displacement magnitude increased. This is illustrated in the $3 \mathrm{D}$ confocal images taken at a fDEP frequency of $20 \mathrm{MHz}$. As depicted, as the voltage increased the low conductive (red) stream covered a greater electrode surface area. This is quantitatively shown - the magnitude of impedance increases with increasing voltage. 
Figure 5. Impedance taken at an excitation frequency of $500 \mathrm{kHz}$ versus applied fDEP frequency for two different interfacial conductivity differences. (a) The magnitude of impedance for varying applied frequencies, $1-20 \mathrm{MHz}$. The interface with a lesser interfacial conductivity has a larger measured impedance. (b) The magnitude of impedance for both systems is rendered dimensionless to compare the two datasets. The impedance measurement is able to non-optically determine that the COF increases with increasing interfacial conductivity. 
(a)

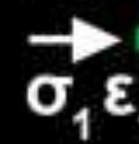

(b) $1 \mathrm{MHz}$ 1 t

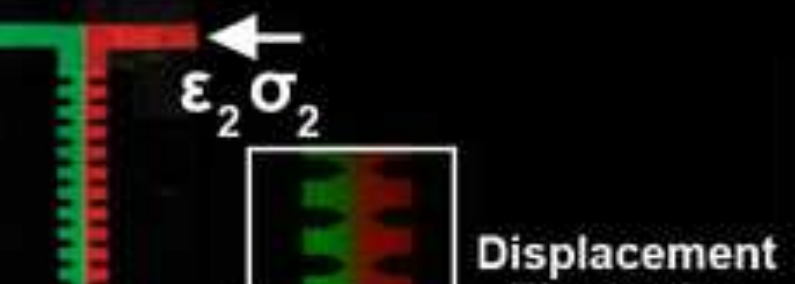
Electrodes

Electrical Interface

$$
\begin{aligned}
& \sigma_{1}>\sigma_{2} \\
& \varepsilon_{1}<\varepsilon_{2}
\end{aligned}
$$

Sensing Electrodes

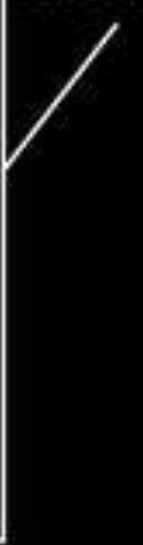

(c) $6.2 \mathrm{MHz}$

(d)

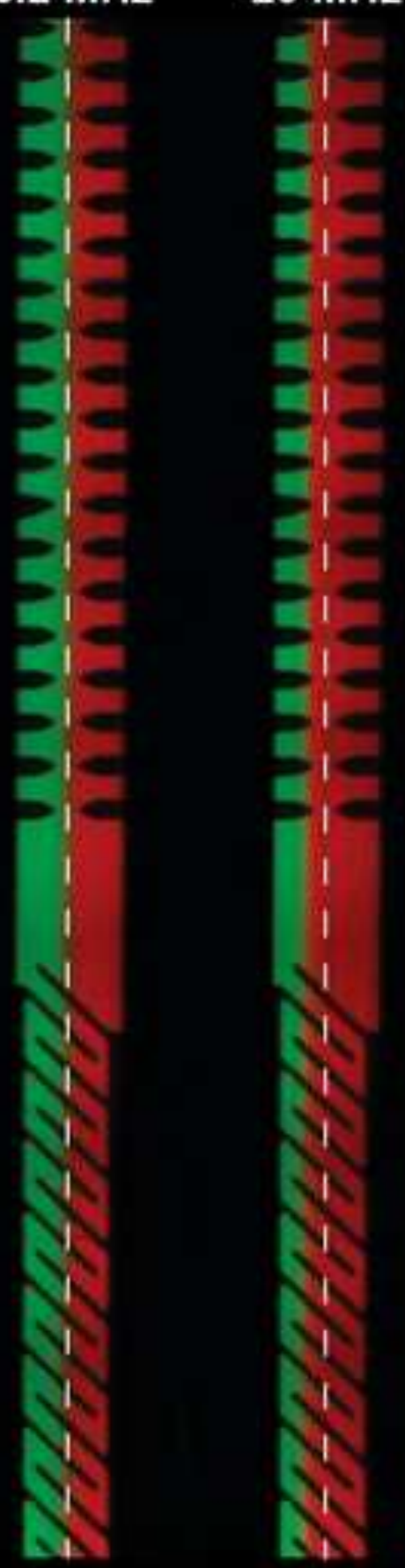


(a) $1 \mathrm{MHz}$

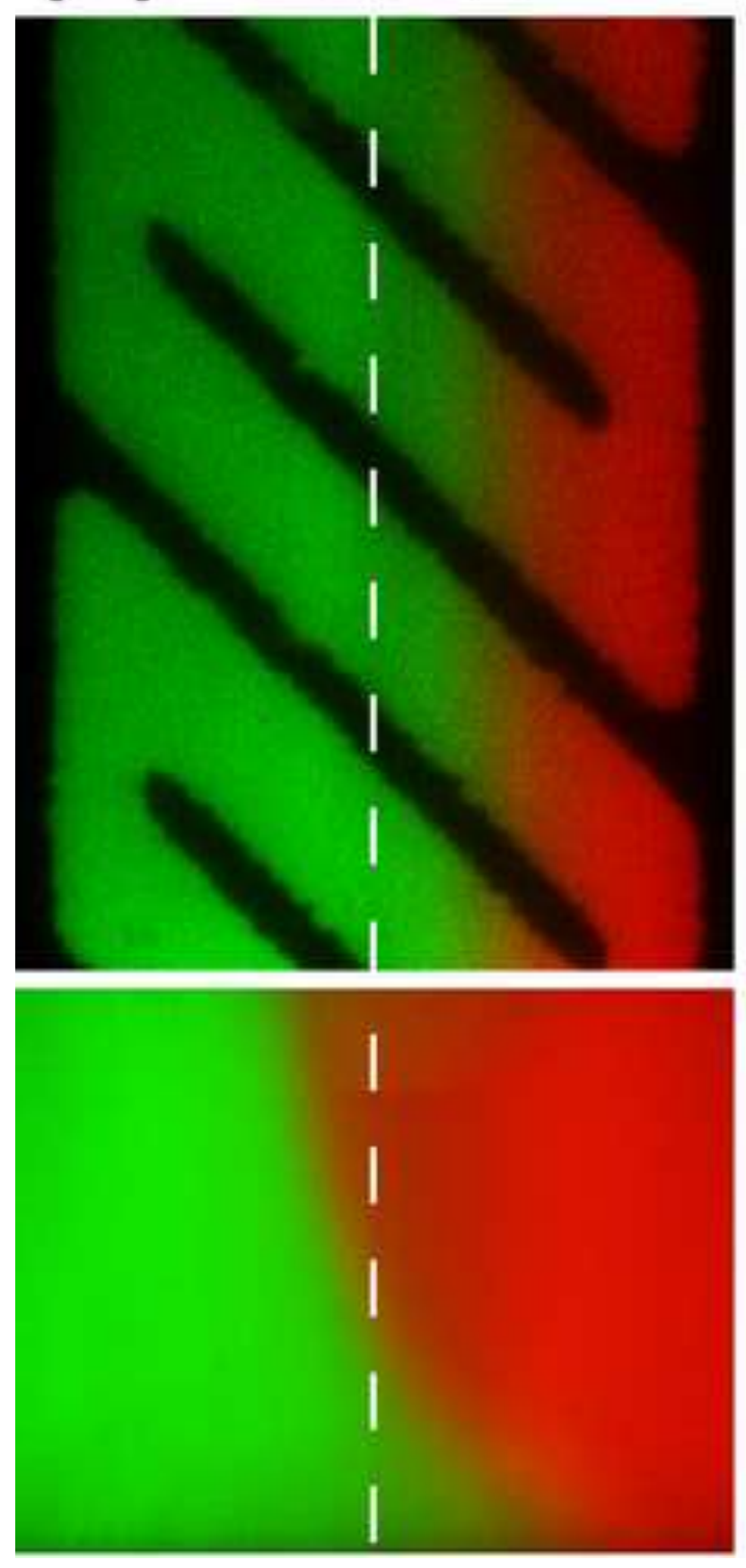

(b) $6.2 \mathrm{MHz}$

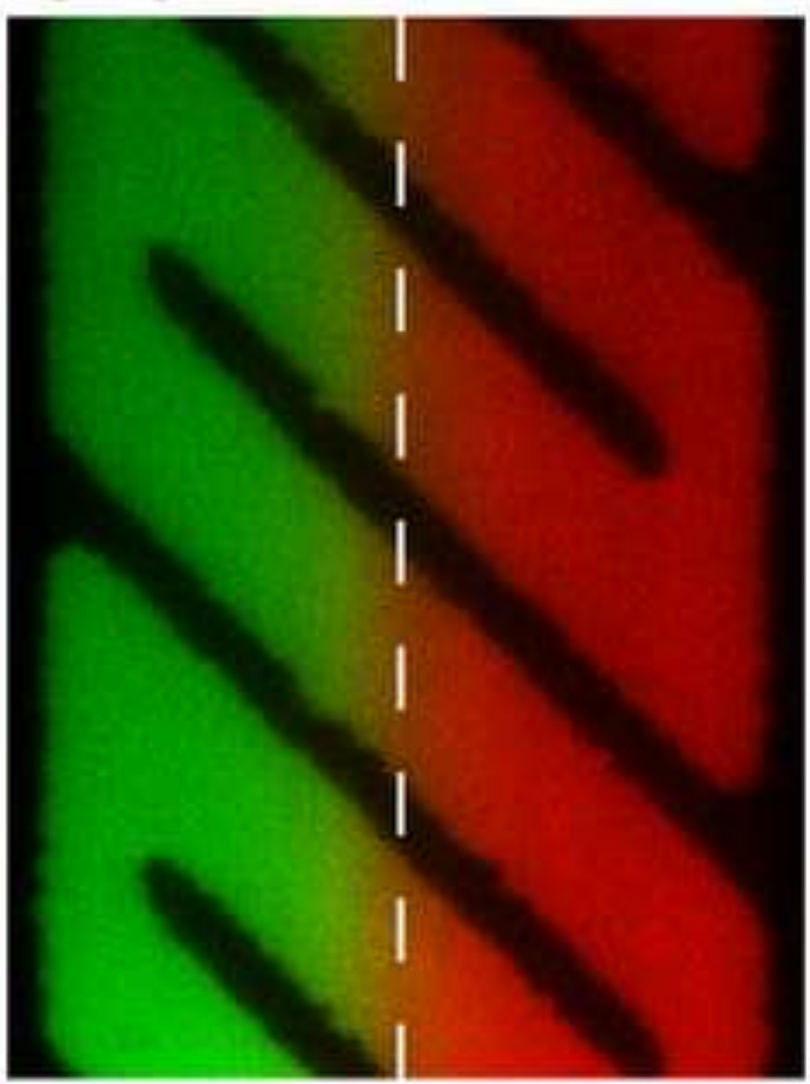

(c) $20 \mathrm{MHz}$

I

I

I

I

I

I

I 50 $\mathrm{m}$

25 jm 


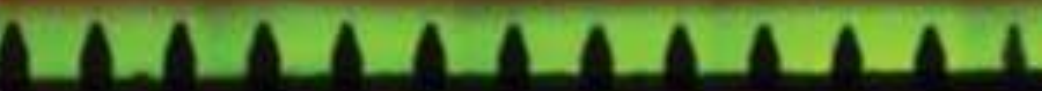

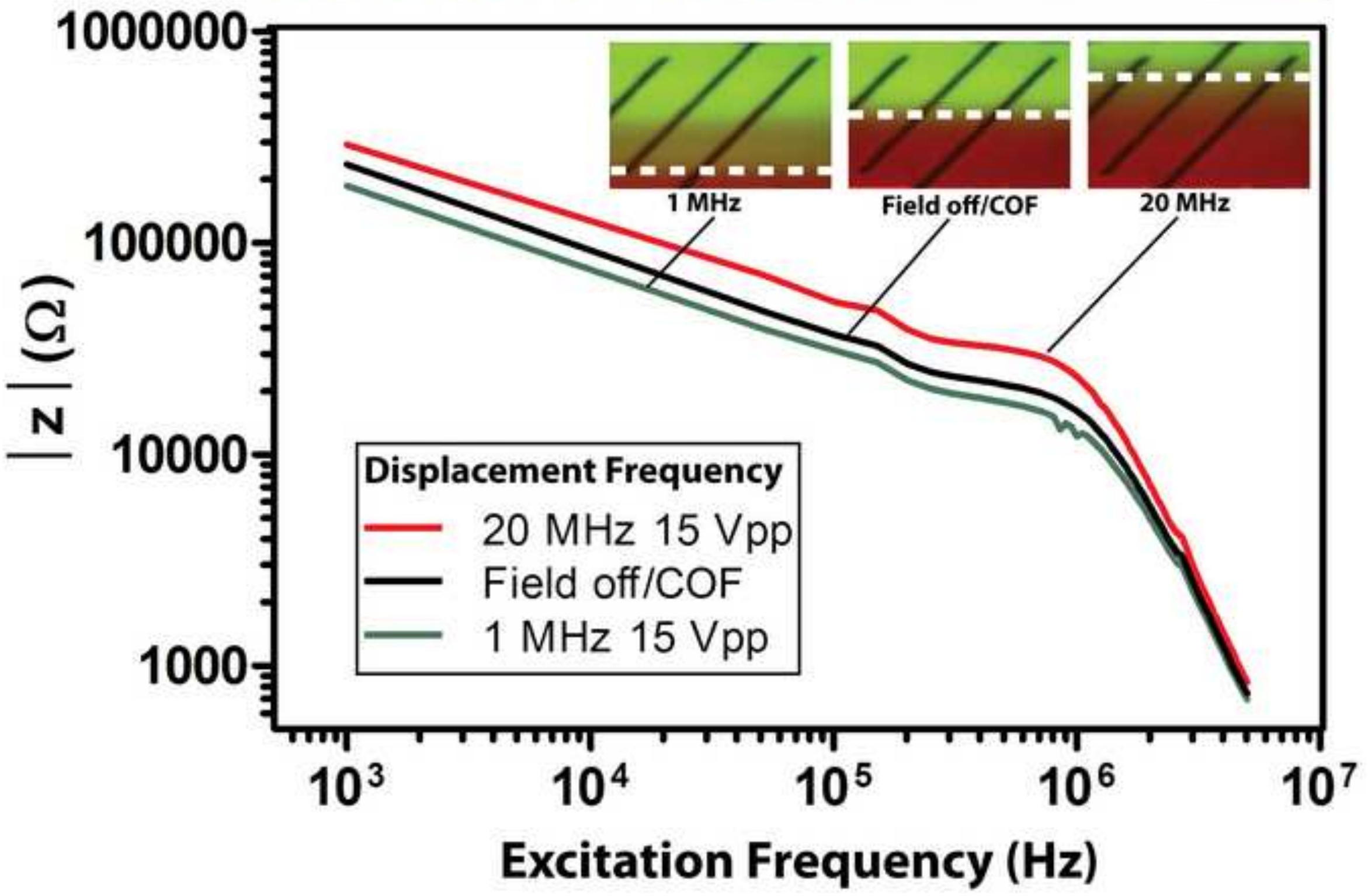




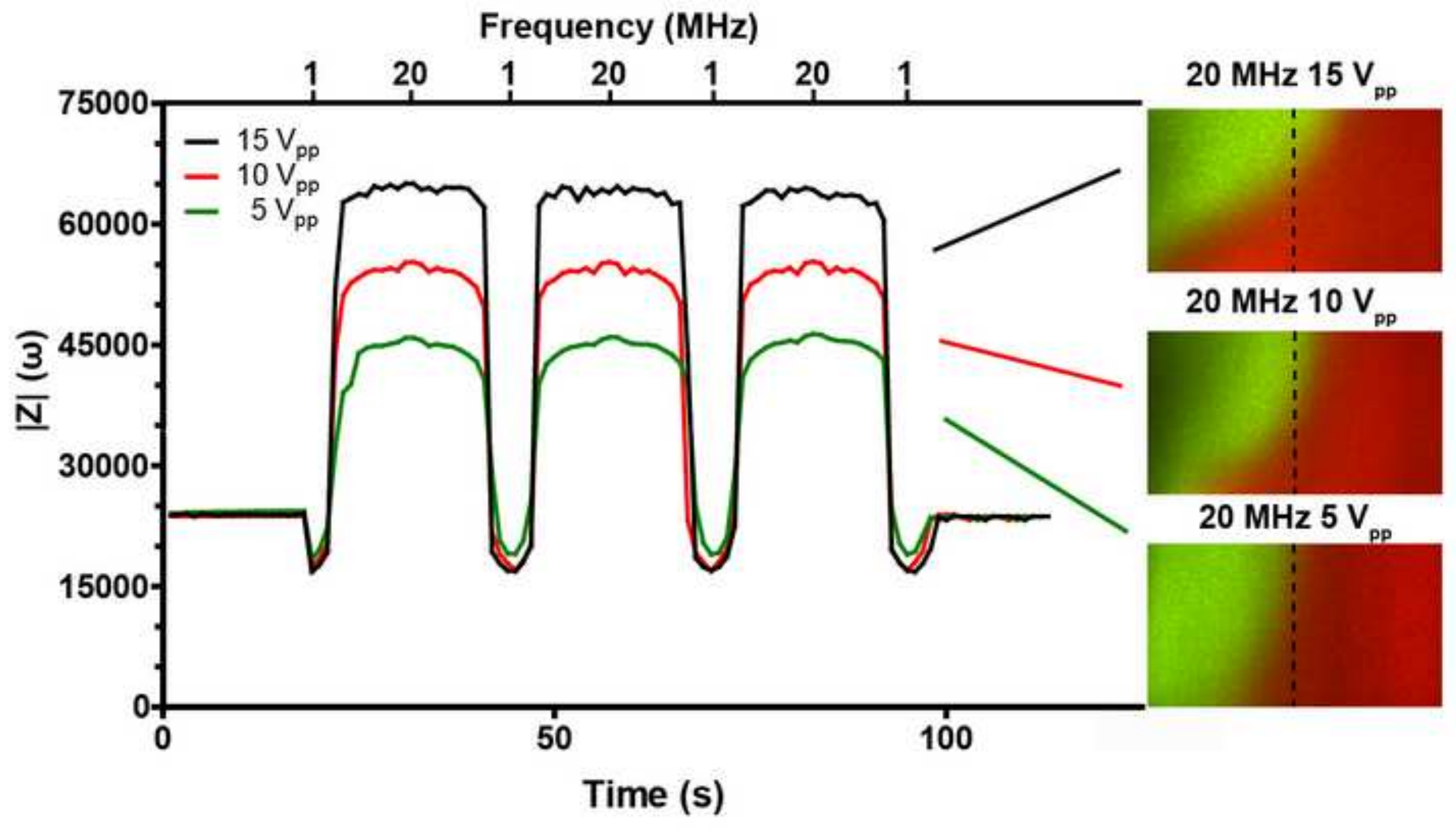


(a)

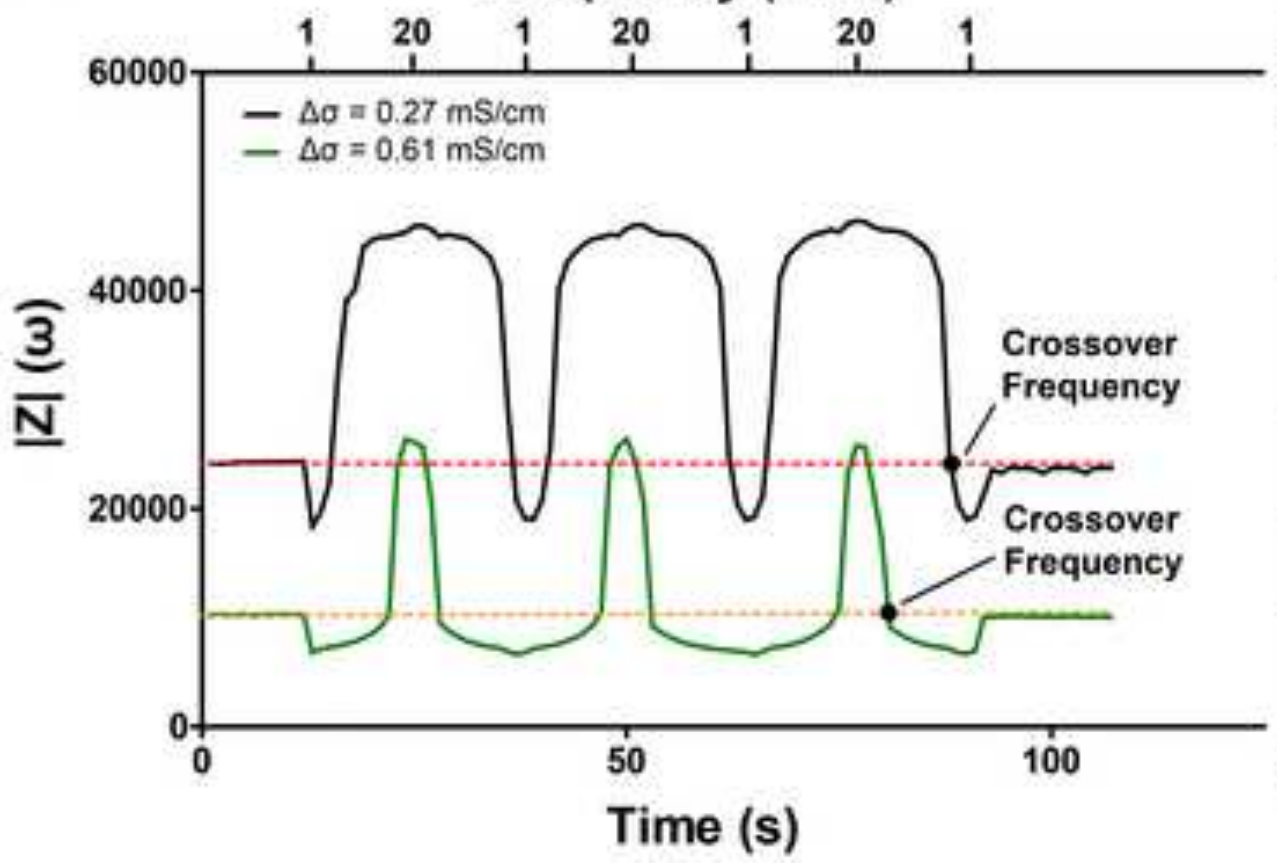

(b)

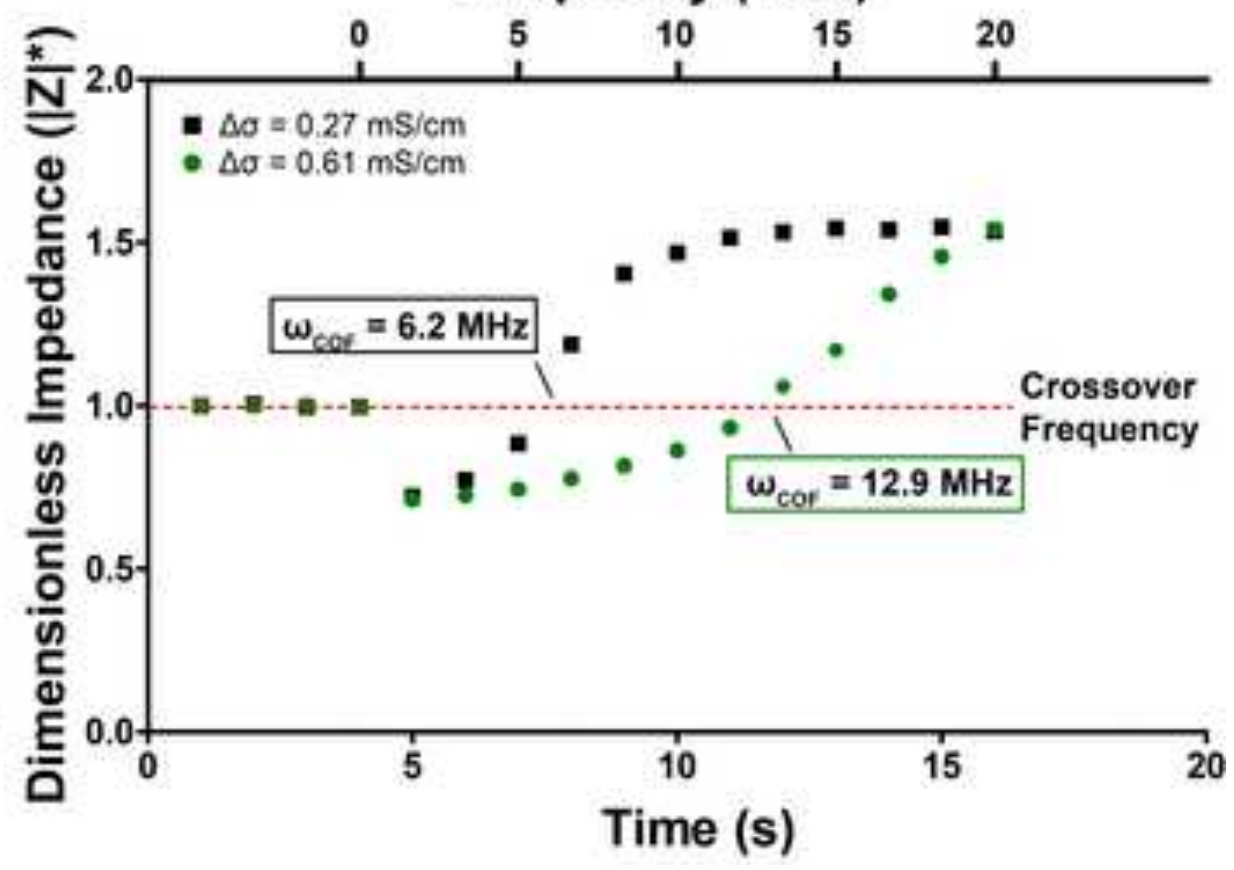

